\title{
Saturation of light- and heavy-hole exciton energies in very narrow quantum wells
}

\author{
Emil S. Koteles and Douglas A. Owens \\ GTE Laboratories Incorporated, Waltham, Massachusetts 02254 \\ Daniel C. Bertolet and Kei May Lau \\ Department of Electrical and Computer Engineering, University of Massachusetts, Amherst, Massachusetts 01003
}

(Received 4 August 1988)

\begin{abstract}
We report the experimental observation of a decrease in the difference in the energies of the ground-state light- and heavy-hole excitons as the widths of single $\mathrm{GaAs} / \mathrm{Al}_{x} \mathrm{Ga}_{1-x} \mathrm{As}$ quantum wells become very narrow. This is a consequence of the saturation of ground-state exciton energies at a value equal to that of excitons in the barrier material. Although this effect is well known theoretically, this is the first clear experimental evidence of a phenomenon which is an additional manifestation of the quantum-mechanical nature of the properties of the structure.
\end{abstract}

The quantization of the energies of excitons spatially confined within a thin layer by potential walls and the increase of these energies as the layer is narrowed are phenomena which have been thoroughly investigated, both theoretically and experimentally, over the last several years. ${ }^{1}$ However, although it has been known theoretically for some time that any quantum well ( $Q W)$ of finite depth, no matter how narrow, must contain at least one bound electronic level (the ground state), no experimental evidence has been reported to date to substantiate this prediction. Experimentally, this is manifested by a maximum, asymptotic, or saturation value for the energy of the ground-state exciton which cannot be exceeded as the width of a quantum well is reduced toward zero. The maximum value is, effectively, equal to the exciton energy in the barrier material. One consequence of this effect is that exciton photoluminescence linewidth broadening due to QW width fluctuations will tend to zero for very narrow wells since changes in the widths of very narrow wells will have little effect on exciton energies which are tending to asymptotic values. This has recently been observed. ${ }^{2}$ The exciton energy saturation effect holds true for both light- (LH) and heavy-hole (HH) excitons in GaAs/ $\mathrm{Al}_{x} \mathrm{Ga}_{1-x} \mathrm{As}$ quantum wells. Thus, for very narrow QW's, ground-state energies for light- and heavy-hole excitons are predicted to converge asymptotically to the same value at zero $Q W$ width. In very wide $Q W$ 's, both of these energies will also converge asymptotically to a value identical to that of bulk GaAs since the spatial confinement effect diminishes in wide wells. Thus, the difference in energy between ground state light- and heavy-hole excitons will be small for wide QW's, will increase as the QW width is decreased (due to spatial confinement effects, i.e., quantization), will reach a maximum value, and then will decrease again in very narrow QW's as light- and heavy-hole exciton energies approach the asymptotic value (equivalent to the ground-state energy of excitons in the bulk barrier material).

Although this effect has been understood theoretically for some time, ${ }^{3}$ the only supporting experimental data up to now has been the observation that, for thin QW's, the light- and heavy-hole exciton energy splitting $\left(\Delta_{\mathrm{LH}}=\mathrm{LH}-\mathrm{HH}\right)$ ceases to increase monotonically in very marrow QW's. ${ }^{4}$ In this paper, we will present experimental evidence to show that $\Delta_{\mathrm{LH}}$ for very narrow QW's $(\sim 1.2 \mathrm{~nm})$ is the same magnitude as for wider QW's $(\sim 8.0 \mathrm{~nm})$ and is as much as three times smaller than the maximum $\Delta_{\mathrm{LH}}$ observed at intermediate $\mathrm{QW}$ widths $(\sim 3.5 \mathrm{~nm})$. This behavior qualitatively follows that predicted by a relatively straightforward calculation based on the model presented above and is conclusive evidence of exciton energy saturation in very narrow $\mathrm{QW}$ 's.

A series of single $\mathrm{GaAs} / \mathrm{Al}_{x} \mathrm{Ga}_{1-x}$ As quantum wells of varying widths were grown on a nominally undoped GaAs substrate using atmospheric pressure organometallic chemical vapor deposition. Growth interruption was employed at each interface in order to improve interface abruptness. Further details of the growth procedure may be found elsewhere. ${ }^{5}$

Light- and heavy-hole exciton energies were experimentally determined in each QW using low-temperature photoluminescence excitation (PLE) spectroscopy which is analogous, especially at low temperatures, to absorption spectroscopy. A photoluminescence spectrum is first taken of each sample. This typically exhibits a series of peaks due to ground-state heavy-hole excitons from each of the QW's. Then, by tuning the spectrometer to the lowenergy side of each $\mathrm{QW}$ photoluminescence peak in turn and fixing it there, the PLE spectrum of each QW is measured separately by monitoring the PL intensity of the QW peak as a function of the energy of the exciting beam. The strongest features in the PLE spectra are due to ground state light- and heavy-hole excitons.

Optical spectra were measured with a double grating spectrometer coupled to a cooled GaAs photomultiplier. Tunable laser excitation energies for the PLE measurements were generated with two dye-laser systems. An Oxazine 750 dye laser pumped with a $\mathrm{Kr}$-ion laser provided wavelengths from 710 to $840 \mathrm{~nm}$ while a DCM dye laser pumped with an Ar-ion laser gave coverage from 610 to 
$690 \mathrm{~nm}$. The samples were mounted, strain-free, in an exchange gas liquid-helium cryostat. The data acquisition systems were controlled by dedicated personal computers.

Low-temperature PL and PLE spectra of three quantum wells of differing widths in one of the two samples studied are presented in Fig. 1. The nominal well widths (as estimated from growth conditions) of the three quantum wells illustrated, QW1, QW2, and QW4, are 1.2, 3.5, and $7.0 \mathrm{~nm}$, respectively. QW3 was omitted from this figure since its energy placed it in the gap between the two dye laser ranges described above so that a complete PLE spectrum could not be measured.

Several features are worth noting. The PL linewidths of the heavy-hole excitons (dashed lines) are narrower than the PLE linewidths of the same transitions. The reason for this difference is not known. The Stokes shift (i.e., the difference in energy between the exciton energy as measured by PL and PLE) increases as the QW width decreases. The exact cause of the Stokes shift is not known but it is believed related to free exciton localization at anomalies (e.g., defects or impurities) at interfaces between well and barrier material. The localization energy is expected to be QW width dependent and so a dependence of the Stokes shift on QW width is not unexpected. ${ }^{6}$ Also, the FWHM's (full width at half maximum) of both the PL and PLE peaks decrease in very narrow wells. This results from the saturation of exciton energies in narrow QW's and is discussed in more detail elsewhere. ${ }^{2}$

The feature, in Fig. 1, of relevance to this discussion is the observation that the difference in energy between the light- and heavy-hole excitons is smaller for the narrowest (QW1) and widest (QW4) quantum wells than for the intermediate well (QW2). This is a direct consequence of the saturation of light- and heavy-hole exciton energies at the $\mathrm{Al}_{x} \mathrm{Ga}_{1-x} \mathrm{As}$ band-gap energy in quantum wells with

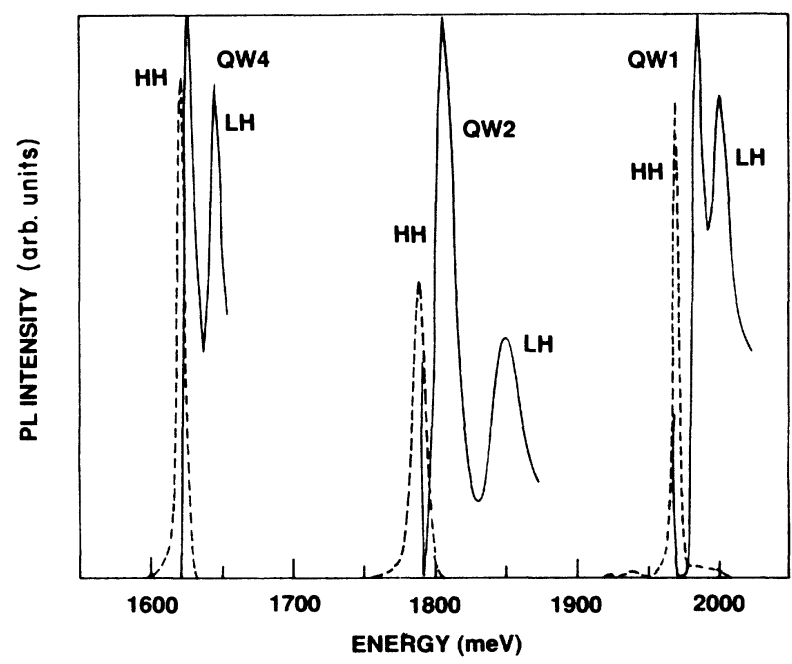

FIG. 1. 5-K PL (dashed lines) and PLE (continuous lines) spectra of a sample containing several QW's of differing widths. The PL peaks and the lower-energy PLE peaks from each QW are due to transitions involving ground-state heavy-hole excitons. The higher-energy PLE peaks in each case are due to light-hole excitons. very narrow widths. In Fig. 2 the data from seven QW's are summarized. Open circles represent experimentally determined splittings of ground-state exciton energies $\Delta_{\mathrm{LH}}$, as a function of nominal $\mathrm{QW}$ widths. The solid line is the prediction of a standard calculation employing commonly used parameters for the $\mathrm{GaAs} / \mathrm{Al}_{x} \mathrm{Ga}_{1-} \mathrm{As}$ system; $m_{e}=0.0665 m_{0}, m_{\mathrm{HH}}=0.34 m_{0}, m_{\mathrm{LH}}=0.092 m_{0}$, and a conduction-band offset equal to $67.5 \%$ of the total band offset. ${ }^{7}$ The total band offset $(523 \mathrm{meV})$ was determined experimentally by taking the difference between the energies of PL peaks due to the bulk GaAs buffer layer and the AlGaAs barrier material. In this model all of the effective masses were assumed to be parabolic with the exception of the electron effective mass. The exciton binding energies were obtained from experiment. ${ }^{8}$ However, since only the difference between the light- and heavy-hole exciton binding energies enters $\Delta_{\mathrm{LH}}$, the calculation is not very sensitive to the exact values of the binding energies.

There is very good qualitative agreement between the calculated curve and the experimental data, although, quantitatively, the agreement is less satisfactory. However, both the calculated curve and the experimental points illustrate the exciton energy saturation effect. As the QW width is reduced, the difference between the light- and heavy-hole exciton energies, $\Delta_{L H}$, first increases (due to spatial confinement effects), goes through a maximum (in this case at around $3 \mathrm{~nm}$ ) and then decreases toward zero. In this series of QW's there is a significant difference (about a factor of three) between the maximum value of $\Delta_{\mathrm{LH}}$, at about $3.5 \mathrm{~nm}$, and the value for the narrowest QW, clearly demonstrating the saturation effect. The unsatisfactory quantitative agreement between experiment and calculation is somewhat surprising since data points obtained from samples grown by molecular beam epitaxy (MBE), in which QW widths were determined by reflection high-energy electron diffraction oscillations, were in excellent agreement with the theory. ${ }^{9}$ This result may be due to subtle differences in the shapes of QW's grown

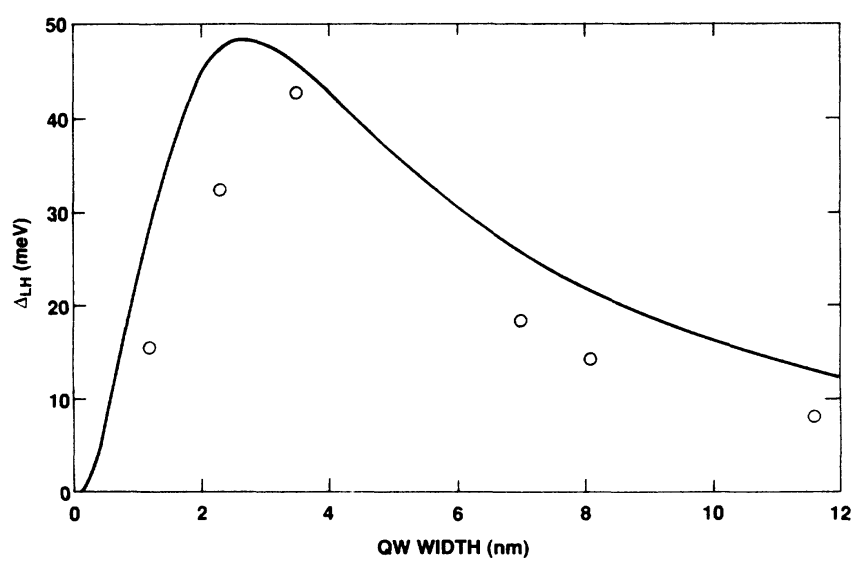

FIG. 2. The difference in the ground-state energies of heavyand light-hole excitons as a function of QW width. The open circles are the experimental values determined by PLE spectroscopy and the continuous line is the calculated dependence using the model described in the text. The vertical size of the circles is comparable to the experimental uncertainty. 
by $\mathrm{MBE}$ and organometallic chemical-vapor deposition (OMCVD) or, more likely, in inaccuracies in determining the width of QW's grown by OMCVD.

In summary, saturation of quantum well excitonic energies at the top of very narrow wells has been demonstrated. The energy splitting between light- and heavy-hole excitons has been experimentally observed to substantially decrease in narrow wells as a consequence of this effect in qualitative agreement with theory. This is the first experimental verification of an effect which has been theoretically predicted for some time and which is an additional demonstration of the quantum mechanical nature of energy levels in semiconductor quantum wells.

${ }^{1}$ For recent reviews on several aspects of quantum wells, see the special issue, Semiconductor Quantum Wells and Superlattices: Physics and Applications [IEEE J. Quantum Electron. QE-22, (1986)].

${ }^{2}$ Daniel C Bertolet, Jung-Kuei Hsu, Kei May Lau, Emil S. Koteles, and Douglas Owens, J. Appl. Phys. (to be published).

${ }^{3}$ See, for example, G. Bastard, Physics and Applications of Quantum Wells, edited by E. E. Mendez and K. von Klitzing, (Plenum, New York, 1987), p. 21.

${ }^{4}$ R. C. Miller, C. W. Tu, S. K. Sputz, and R. F. Kopf, Appl.

Phys. Lett. 49, 1245 (1986).

${ }^{5}$ Daniel C. Bertolet, Jung-Kuei Hsu, and Kei May Lau, J. Appl. Phys. 62, 120 (1987).

${ }^{6}$ L. Vina, R. T. Collins, E. E. Mendez, and W. I. Wang, Phys. Rev. B 33, 5939 (1986).

${ }^{7}$ See, for example, G. Duggan, H. I. Ralph, and K. J. Moore, Phys. Rev. B 32, 8395 (1985).

${ }^{8}$ Emil S. Koteles and J. Y. Chi, Phys. Rev. B 37, 6332 (1988).

${ }^{9}$ Emil S. Koteles and B. Elman (private communication). 